\title{
Application of a graded screen array for size measurements of radioactive aerosols in accelerator rooms
}

\author{
By N. Osada ${ }^{1, *}$, Y. Oki ${ }^{2}$, H. Kanda ${ }^{1}$, K. Yamasaki ${ }^{2}$ and S. Shibata ${ }^{2}$ \\ ${ }^{1}$ Graduate School of Engineering, Kyoto University, Katsura, Nishikyo-ku, Kyoto 615-8530, Japan \\ 2 Research Reactor Institute, Kyoto University, Kumatori, Osaka 590-0494, Japan
}

(Received December 22, 2009; accepted in revised form November 11, 2010)

\begin{abstract}
Radioactive aerosols / Particle size distribution / Graded screen array / Radioactive gas / Accelerator / Radiation protection
\end{abstract}

\begin{abstract}
Summary. A rapid measurement method for aerosol particle size is required to clarify the formation mechanism of freshlyformed radioactive aerosols in accelerator rooms. A graded screen array (GSA) method only requires brief sampling. Therefore, the GSA method is suitable for the measurement of radioactive aerosols in the accelerator rooms. In this work, a conventional GSA was applied to measure the particle size distribution of an aerosol. An influence of the radioactive gas on the GSA measurement was found. The adsorption of radioactive gases resulted in a discrepancy between the results of the GSA method and those of the diffusion battery method. An improved GSA method was developed to measure the radioactive aerosol formed in the accelerator room. The adsorption was measured by the improved GSA, and the influence of the radioactive gas was eliminated. The result of the improved GSA showed fair agreement with that of the diffusion battery system.
\end{abstract}

\section{Introduction}

High-energy and large-current accelerators have been recently developed. In accelerator rooms, the primary beams in the air and the strong secondary radiation intensify the nuclear and chemical reactions that are occurring in the air. These reactions result in the production of high concentrations of radioactive gases and aerosols. This radiationchemical process of aerosol formation can be compared with processes in the earth's atmosphere. High-energy cosmic rays may cause the dissociation of air molecules and the formation of particles at high altitudes. The products of such a reaction form particles that are able to induce the formation of clouds [1,2]. From the point of view of radiation protection of workers in accelerator facilities, the inhalation of radioactive aerosols, such as radon daughter aerosols, in the accelerator room may cause internal radiation exposure. Information on radioactive aerosols is important to evaluate the risk of such internal exposure. Whereas aerosols in the environment have many sources (natural and artificial) that are hard to identify, the source of aerosols in

\footnotetext{
*Author for correspondence (E-mail: osada@HL.rri.kyoto-u.ac.jp).
}

the accelerator room is simply the chemical reaction of the air molecules caused by irradiation. The aerosol particles are observable in the early phase of their formation and growth. Freshly-formed particles are nanometer sized, and their size and concentration change rapidly. Many measurement methods for radioactive aerosols have been previously developed. The diffusion battery (DB) [3], the inertial impactor and the graded screen array (GSA) [4] have been used for the measurement of radioactive aerosols such as those of radon daughters. It is thought that these methods could also be used for the measurement of accelerator aerosols. However, the diffusion battery method often requires a long measurement time, i.e., it is unable to measure changes in the size distribution of freshly-formed accelerator aerosols. Parallel diffusion batteries can make measurements rapidly, however, large systems and samples of irradiated air are required. The impactor method has low size resolution for the nano-sized accelerator aerosols. Aged stable accelerator aerosols of larger particles have been measured with a diffusion battery and an impactor. In this study, the influence of a radioactive gas on aerosol measurements in an accelerator room was analyzed by the GSA method. The GSA was applied to measure the freshly-formed aerosols in an accelerator room. The GSA enabled rapid measurement and required only a small amount of sample air. The results of the DB and GSA were compared. Interference was observed within the measurements made by the GSA method. The reason for the interference was elucidated, and a new GSA method was developed to overcome the interference.

\section{Experimental}

Irradiation experiments were performed at the $46-\mathrm{MeV}$ electron linear accelerator in the Research Reactor Institute of Kyoto University. Radioactive and non-radioactive aerosols were formed in the accelerator room of this accelerator. An accelerated electron beam bombarded a tantalum target in water to produce neutrons and bremsstrahlung. The electrons were accelerated to $30 \mathrm{MeV}$. The electron current was $100 \mu \mathrm{A}$. An irradiation chamber was placed so tha it crossed the beam line at a distance of $30 \mathrm{~cm}$ from the target (Fig. 1). The chamber was a stainless steel tube with an inner diameter of $50 \mathrm{~mm}$. Aerosol-free sample air was produced with 


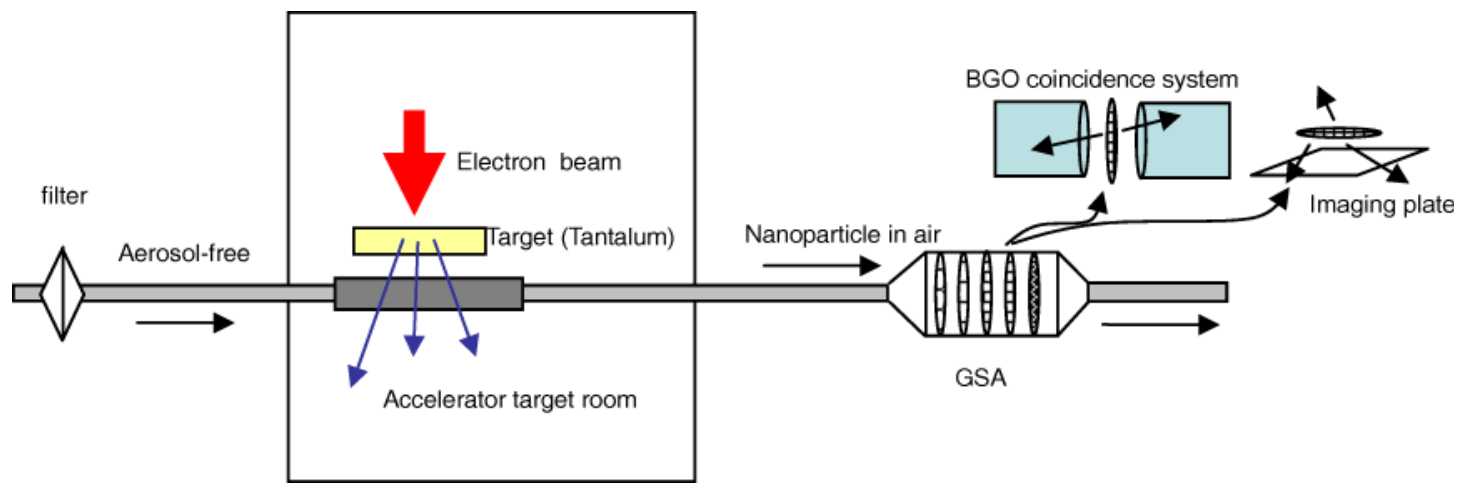

Fig. 1. Experimental setup of the irradiation and measurement system. After sampling the aerosol, the activities on the screens and on a filter were measured by a BGO coincidence system or an imaging plate.

a cartridge HEPA filter. The absence of the aerosol in the air was verified with a condensation particle counter (CPC, Model 3025A-S or 3022, TSI Inc.) The sample air was transported to the irradiation chamber in the accelerator room and subjected to irradiation. By the aid of a tube, the irradiated air was transferred to a measurement system installed in the room neighboring the accelerator room. This tube-type irradiation setup supplied particles that were of similar age and had stable particle size distributions.

Non-radioactive aerosols were measured with both a differential mobility analyzer (DMA, Model 3070, TSI Inc.) and a CPC. The results were compared with those of the DB method. The measurement system for radioactive aerosols consisted of a DB and a GSA. The aerosol was conducted with the DB and the GSA at a sample airflow rate of $4.5-25.5 \mathrm{~L} / \mathrm{min}$. The flow rates were adjusted with massflow controllers. The concentration of the formed activity in the irradiated air was measured with an ionization chamber. Both the total activity of the air and the truly gaseous activity of the air (as obtained by filtering the aerosols from the air) were determined.

A high-purity germanium semiconductor detector and a coincidence system consisting of two bismuth germanate (BGO) scintillation detectors were applied to measure the activity on the membrane filters. The data obtained by the $\mathrm{CPC}, \mathrm{Ge}$ and BGO detectors were recorded by connected computers. Radioactive nuclides were identified and determined with the Ge detector. The annihilation photons were determined from the ratio of the activity of the $\beta^{+}$decay nuclides using the BGO detectors.

\subsection{Theory of the diffusion battery and the graded screen array}

A screen-type diffusion battery (DB) and a graded screen array (GSA) were used for measuring the size distribution of the aerosol particles. The DB and the GSA consisted of stacks of wire screens in a tube. The screens of the DB were made of stainless steel wire of 500 mesh and 500 wires per inch. In this experiment, the DB consisted of seven tubes. The numbers of screens contained in the tubes of the DB were $0,1,2,5,10,20$ and 40 . The GSA consisted of single screens of 30, 100, 300, 500 and 635 mesh. The diameter of the wire screens was $47 \mathrm{~mm}$. For small particles, the penetration through the DB depended upon Brownian diffusion.
Diffusion of particles is the dominant process of particle collection for nanoparticles. The diffusion coefficient can be expressed as a function of particle size. Effects of inertia, gravitational settling and interception are negligible for nanoparticles. Particles that penetrated through the DB were collected on a membrane filter (the collection efficiency was over $99.999 \%$ ). The efficiency was checked by a CPC. The activity of the particles collected on the membrane filter (PTFE filter, ADVANTEC) was measured for the case of use of the DB method, while in the case of use of the GSA method the activity on the screens was determined.

The size distribution of the aerosol particle was assumed to be a lognormal distribution. The ratio of the number concentrations of penetrating particles through the DB and GSA was expressed by an integral of the penetration ratio multiplied by the size distribution. The geometric mean diameter (GMD) and the geometric standard deviation (GSD) were derived from the penetration ratio by a least-squares fit using the following equations (cgs).

$$
\begin{aligned}
P= & \frac{N}{N_{0}}=\frac{A}{A_{0}}=\int_{-\infty}^{\infty} \exp \left\{-K N_{s}\left(\frac{Q}{D(r)}\right)^{\frac{3}{2}}\right\} \\
& \times \frac{1}{\sqrt{2 \pi} \log \sigma_{\mathrm{g}}} \exp \left\{-\frac{\left(\log r-\log r_{\mathrm{g}}\right)^{2}}{2 \log ^{2} \sigma_{\mathrm{g}}}\right\} \mathrm{d} \log r,
\end{aligned}
$$

where $P$ is the penetration ratio, $N$ and $N_{0}$ are the penetrated and the initial number concentrations of the particle, respectively, and $A$ and $A_{0}$ are the penetrated and the initial activity of the particles (cps), respectively. The reason that the ratio of the number concentration is expressed as the activity is described in the results and discussion section. $K$ is the specific number of the screen, $N_{\mathrm{s}}$ is the number of the screen, $Q$ is the air flow rate $(\mathrm{L} / \mathrm{min}), r_{\mathrm{g}}$ is the geometric mean radius (GMR, $\mathrm{cm}$ ) and $\sigma_{\mathrm{g}}$ is the geometric standard deviation (GSD).

$D(r)$ is represented by

$$
D(r)=\frac{\kappa T}{6 \pi \eta r}\left\{1+A \frac{L}{r}+C \frac{L}{r} \exp \left(-\frac{B r}{L}\right)\right\}
$$

where $\kappa$ is the Boltzmann constant, $T$ is the temperature of the air $(\mathrm{K}), \eta$ is the viscosity of the air $\left(\operatorname{dyn~s} / \mathrm{cm}^{3}\right), L$ is the mean free path of the air molecule and $r$ is the radius of the aerosol particle (cm). $A, B$ and $C$ are the coefficients 
for the Cunningham correction $(A=1.246, B=0.87$ and $C=0.42)$ [5]. The values of $r_{\mathrm{g}}$ and $\sigma_{\mathrm{g}}$ were derived from the penetration ratio which was experimentally obtained by a least-squares fit using Eqs. (1) and (2).

Irradiated sample air was introduced into each DB for $10 \mathrm{~min}$. After a sampling period of approximately $1 \mathrm{~min}$, the measurement with the BGO system was started. The DB measurement required approximately $100 \mathrm{~min}$. The sampling duration for the GSA was $20 \mathrm{~min}$. An imaging plate (BAS-2025, Fujifilm corp.) was exposed to the radiation from the radionuclides on the screens of the GSA for $20 \mathrm{~min}$ after the 5-min sampling was finished. The linearity of the signals from the imaging plate was verified using a standard gamma source. The latent image of the imaging plate was converted into digital images by an imager. Four square centimeters of the image of the center of the wire screens were defined as the region of interest. The activity was calculated from the image of the region of interest. Using the obtained concentration of activity, $r_{\mathrm{g}}$ and $\sigma_{\mathrm{g}}$ were derived from the penetration ratio using a similar fit as that of Eq. (1). To measure the activity of the adsorption of the radioactive gas on the screens of the GSA, the irradiated air was branched and conducted to two GSAs. A pre-filter that collected particles was inserted upstream of the second GSA. The first GSA collected both radioactive aerosols and gas, and the second GSA collected only radioactive gas.

\section{Results and discussion}

The irradiated air containing radioactive particles and gas was measured with an ionization chamber. The concentration and the percentage of the activity formed in the irradiated air were derived from the decay curve of the activity. The isotopes that formed in the irradiated air were assumed to consist of nuclides [6], ${ }^{15} \mathrm{O},{ }^{13} \mathrm{~N}$ and ${ }^{41} \mathrm{Ar}$. The respective percentages of the three radionuclide activities in the air were found to be $50 \%, 46 \%$ and $4.7 \%$, based on a leastsquares fitting of the decay curve (Fig. 2). The activity of the particles collected on the filter was measured by the Ge semiconductor detector and the BGO coincidence system. The percentage of ${ }^{13} \mathrm{~N}$ was found to be approximately $95 \%$ by the decay analysis of the annihilation peak (Fig. 3). In this accelerator, ${ }^{13} \mathrm{~N}$ was mainly found in the freshly formed radioactive particles. The particles did not included ${ }^{15} \mathrm{O}$ nuclides, which comprised a large content of the air and war-

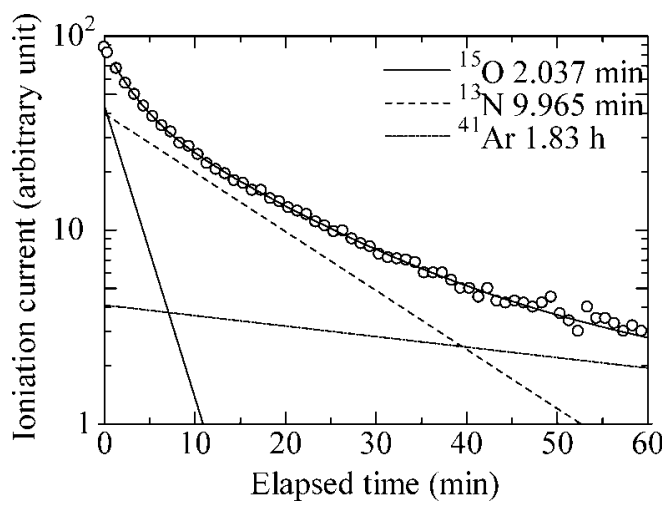

Fig. 2. Change in the ionization current from the irradiated air.

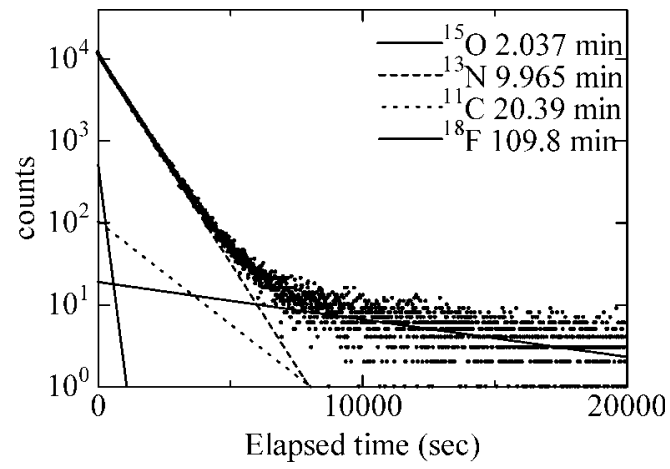

Fig. 3. Change in the activity of the membrane filter.

rants future study. The freshly-formed radioactive aerosols were considered to only consist of ${ }^{13} \mathrm{~N}$ radioactive particles in this study.

The total concentration of the aerosol particles formed was measured with a CPC that counted particles using a laser. The concentration was found to be $6.0 \times 10^{6}$ particles $/ \mathrm{cm}^{3}$ at the sample air flow rate of $10 \mathrm{dm}^{3} / \mathrm{min}$. The radioactive nuclides in the air were formed at a low concentration, approximately $7 \mathrm{~Bq} / \mathrm{cm}^{3}$. The irradiated air contained over $10^{6}$ particles and approximately $7 \mathrm{~Bq}$. The formed particles were almost all non-radioactive, and few particles were ${ }^{13} \mathrm{~N}$-radioactive aerosols. The possibility that one particle contained several radioactive atoms was negligibly small. Therefore, the size distribution was calculated using Eq. (1) by substituting the ratio of the activity of the aerosol particles, $A / A_{0}$, for the ratio of the number concentration, $N / N_{0}$, in Eq. (1). This assumption was applied to the analysis of the measurement of the DB and the GSA.

Fig. 4 shows the result of a measurement of the mean particle diameter of non-radioactive aerosol with the DB and the DMA. The DMA has been widely used for particle size measurements of aerosol particles. The dependence of the particle size on the irradiation time was observed. The tendency toward long-time irradiation to increase the particle size has been reported from an irradiation experiment using an alpha source was well as within an operating accelerator room $[7,8]$.our results using DMA were almost the same as the results in references 7 and 8 . The result of the DMA system showed fair agreement with that of the DB system. Both the DB and the DMA were successfully employed for the measurement of the particle size distribution.

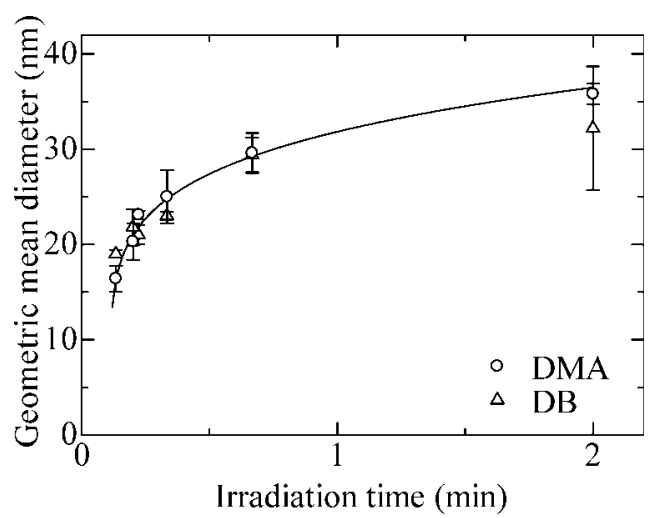

Fig. 4. Comparison of the analyzed geometric mean diameter of the non-radioactive aerosol analyzed by DMA and DB. 


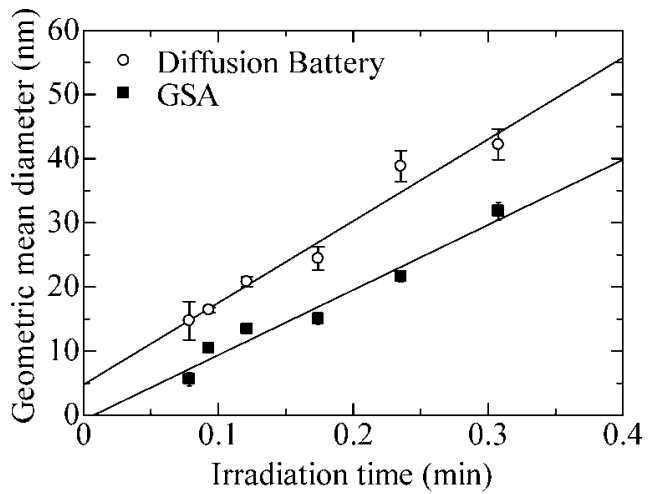

Fig. 5. Relationship between irradiation time and geometric mean particle size of ${ }^{13} \mathrm{~N}$ radioactive aerosols as analyzed by DB and GSA.

Fig. 5 yields results of measurements by the DB and the GSA. The diameter obtained with the GSA was smaller than that found by the DB. The DB method showed a dependence of the particle diameter on the flow rate of the sample air. The result of the DB measurement revealed the same dependence of the size distribution in the ${ }^{13} \mathrm{~N}$-radioactive aerosol as in the non-radioactive aerosol. However, did the results of the GSA shift toward lower values? The measurement of radioactive aerosols with the GSA method in the accelerator room was influenced by the presence of coexisting radioactive gases. It is assumed that the radioactive gas condensed on the screens of the GSA. Radioactive ${ }^{13} \mathrm{~N}$ atoms are thought to attach to the surface of the wire of the screens by adsorption of the radioactive gas. This adsorption shifted the obtained diameters to smaller ones in the GSA measurement.

The activity ratio between the radioactive particles and the radioactive gas in the irradiated air was measured with an ionization chamber to be 1:9. This result means that radioactive gas in addition to the radioactive particles was formed in the accelerator room. In our interpretation of the data, it was assumed that the measured activity indicates the number of radioactive particles when radioactive particles were sampled by the GSA. However, although a small percentage of the condensed radioactive gas adsorbed to the particle collector of the GSA, there was a strong influence of the radioactive gas on the measurement.

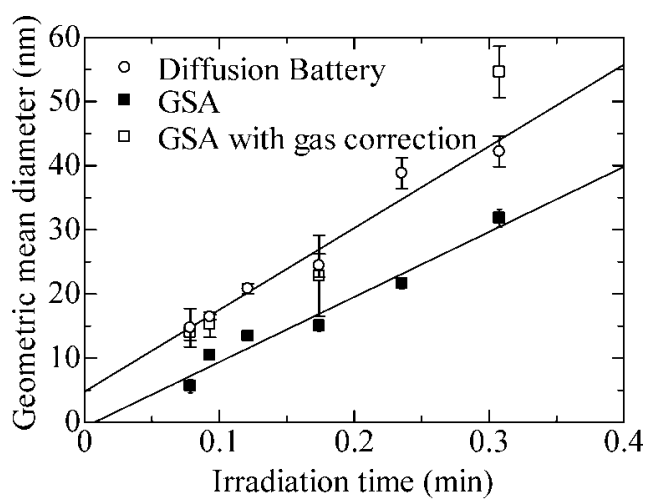

Fig. 6. Relationship between irradiation time and geometric mean particle diameter of ${ }^{13} \mathrm{~N}$ radioactive aerosols as determined by DB, GSA and GSA methods with gas correction.
Therefore, the amount of the condensed radioactive gas on the screens was measured. The activity of the radioactive gas adsorbed on the screens was approximately twice the activity of the radioactive particles. This gas measurement was carried out in the conventional single GSA arrangement. The result of the GSA method was improved by considering the measurement of gas (Fig. 6). The correction concerning the gas adsorption made both $r_{\mathrm{g}}$ and $\sigma_{\mathrm{g}}$ larger. The particle size distribution corrected for the influences of the gas approached the distribution measured by the DB. Without the correction, the activity on the screens was interpreted as resulting from the adsorption of the radioactive gas. The activity on the screens was interpreted to be caused by collected particles, i.e., the activity of the adsorbed radioactive gas was thought to be small radioactive aerosol particles.

\section{Conclusion}

The aerosols formed in irradiation facilities have been difficult to measure. The diameter of the freshly-formed aerosols changes quickly. The diffusion battery and impactor have been widely used to study such phenomena. However, these methods are unable to measure many features because the DB is time consuming, and the effective measuring range of the impactor is large for freshly-formed particles. The conventional single GSA method often does not work properly for the size measurement of radioactive aerosols in the accelerator room of an electron linear accelerator. We found that co-existing radioactive gases influenced the particle sizes obtained with the GSA. We suggest that the adsorption of co-existing radioactive gas caused the discrepancy between the GSA and diffusion battery methods. To compensate for this influence, an additional GSA set was employed to estimate the percentage of adsorption of radioactive gas. The effect of adsorption of the gas was successfully eliminated through this approach. The distribution of the particle sizes measured with the GSA approached the distribution according to DB measurements. An improved GSA method using two GSA sets enabled a quick measurement and required a smaller amount of air than the DB method. It is expected that this new GSA method will help reveal the mechanism of the formation of aerosol particles, especially radioactive aerosols. Measurement using this new GSA in irradiation facilities will be useful to study the radiation protection and will enable us to estimate the particle sizes that dominate attachment of the radioactive particles to the lungs of the workers [9] when an accelerator is operational.

\section{References}

1. Larslaw, K. S., Harrison, R. G., Kirkby, J.: Cosmic rays, clouds, and climate. Science 298, 1732 (2002).

2. Harrison, R. G., Stephenson, D. B.: Empirical evidence for a nonlinear effect of galactic cosmic rays on cloud. Proc. R. Soc. A 462, 1221-1233 (2006).

3. Cheng, Y. S., Yeh, H. C.: Theory of a screen-type diffusion battery. Aerosol Sci. 11, 313-320 (1980).

4. Fukutsu, K., Yamada, Y., Tokonami, S.: Newly designed graded screen array for particle size measurements of unattached radon decay products. Rev. Sci. Instrum. 75(3), 783-787 (2004).

5. Demarcus, W., Thomas, J.: ORNL-1431 (1952).

6. Endo, A., Oki, Y., Kanda, Y., Oishi, Y., and, Kondo, K., Evaluation of internal and external doses from ${ }^{11} \mathrm{C}$ produced in the air 
in high energy proton accelerator tunnels. Radiat. Prot. Dosim. 93(3), 223-230 (2001).

7. Kim, C. S., Adachi, M., Okuyama, K., Seinfeld, J. H.: Effect of $\mathrm{NO}_{2}$ on particle formation in $\mathrm{SO}_{2} / \mathrm{H}_{2} \mathrm{O} /$ air mixtures by ioninduced and homogeneous nucleation. Aerosol Sci. Tech. 36, 941-952 (2002).
8. Yokoyama, S., Sato, K., Manabe, K., Noguchi, H., Kaneko, H., Oki, Y., Iida, T., Tanaka, Su.: Ratio of aerosol and gases of radioactive chlorine and particle size distribution of aerosol formed by high-energy proton irradiation. Radia. Prot. Dosim. 1-6 (2007).

9. ICRP: Dose Coefficients for Intakes of Radionuclides by Workers. ICRP publication 68. Ann. ICRP 24(4) (Oxford Elsevier Science). 